\title{
Glycolipid Polymer Synthesized from Natural Lactonic Sophorolipids by Ring-Opening Metathesis Polymerization
}

Wei Gao, Rena Hagver, Vishal Shah, Wenchun Xie, Richard A. Gross ",M. Firat Ilker, Chrissy Bell, Kelly A. Burke, E. Bryan Coughlin

$$
\text { Supporting information: }
$$

\section{LC-MS method for sopholipids:}

A Waters ZQ LC/MS system was used for analysis and identification of sophorolipids. The system includes 2795 Alliance HT Separation Module, 2996 Photodiode Array Detector and Waters (Micromass) ZQ 2000 on-line Mass Detector. Separation was carried out on a $250 \times 4.6$ mm polymer reversed phase PLRP-S column, with particle size $8 \mu \mathrm{m}$ and pore size $1000 \AA$ (Polymer Laboratories). The mobile phase consisted of water/acetonitrile and was programmed as follows: $60 \%$ water to $50 \%$ water in $20 \mathrm{~min}$, then $60 \%$ water for $5.0 \mathrm{~min}$ (re-equilibration). The HPLC eluent $(1 \mathrm{~mL} / \mathrm{min})$ was split at the outlet of the column by means of a zero-deadvolume tee splitter to introduce eluent to PDA detector and Mass spectrometer. LC-MS was operated using an Atmospheric Pressure Chemical Ionization (APCI) probe in positive ion mode. Probe and source temperature were set to 450 and $50{ }^{\circ} \mathrm{C}$, respectively. Cone voltage was set to $60 \mathrm{~V}$ and corona current to $0.66 \mu \mathrm{A}$. The nebulizer gas flow was set to $300 \mathrm{~L} / \mathrm{h}$. Sophorolipids were detected in scan mode, scan duration $2.0 \mathrm{sec}$, inter-scan delay $0.1 \mathrm{sec}$, and scan range $170-850 \mathrm{~m} / \mathrm{z}$. Data acquisition and mass spectrometric evaluation were performed by use of MassLynx-Software 4.0. The samples for LC/MS were dissolved in 50:50 acetonitrile/water at a concentration of $40-200 \mu \mathrm{g} / \mathrm{mL}$, and filtered through a $0.45 \mu \mathrm{m}$ PTFE filter before injection. 


\section{Lactonic Sophorolipids}
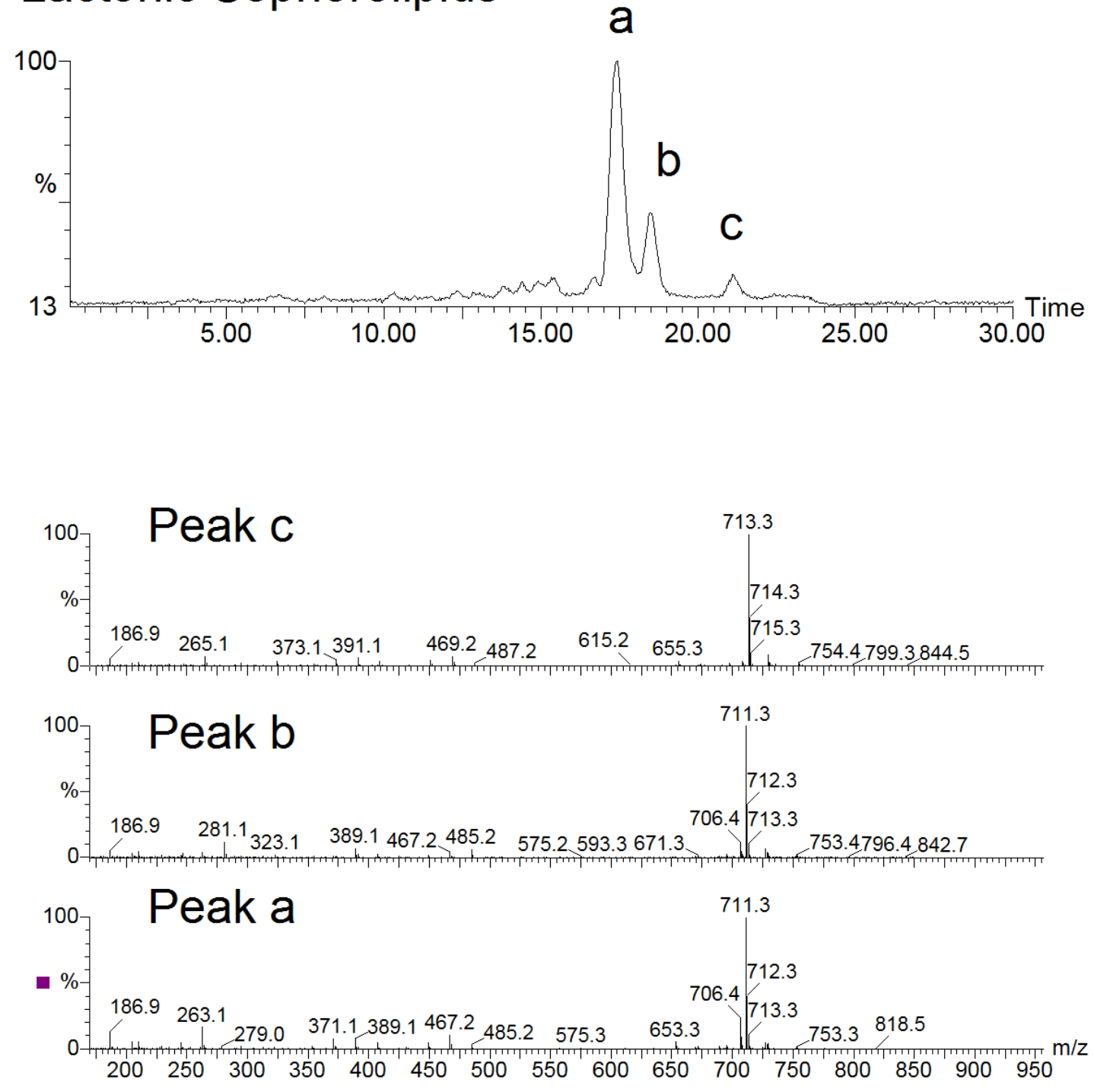

Figure S-1a. Total Ion chromatograph (TIC) of purified lactonic form sophorolipids (top), and mass spectra of major peaks, a, b and c (bottom). Peaks a and b are assigned to the structural isomers 17-L-([2'-O- $\beta$-D-glucopyranosyl- $\beta$-D-glucopyranosyl]oxy)-9-octadecenoic acid 1'-4'”lactone 6',6',-diacetate and 18-L-([2'-O- $\beta$-D-glucopyranosyl- $\beta$-D-glucopyranosyl]oxy)-9octadecenoic acid 1'-4"'-lactone 6',6"'-diacetate. Peak c is assigned to the saturated form of a, that is 17-L-([2'-O- $\beta$-D-glucopyranosyl- $\beta$-D-glucopyranosyl]oxy)-octadecanoic acid 1'-4'”lactone 6', 6',-diacetate. 


\section{Residual from ROMP of SL}
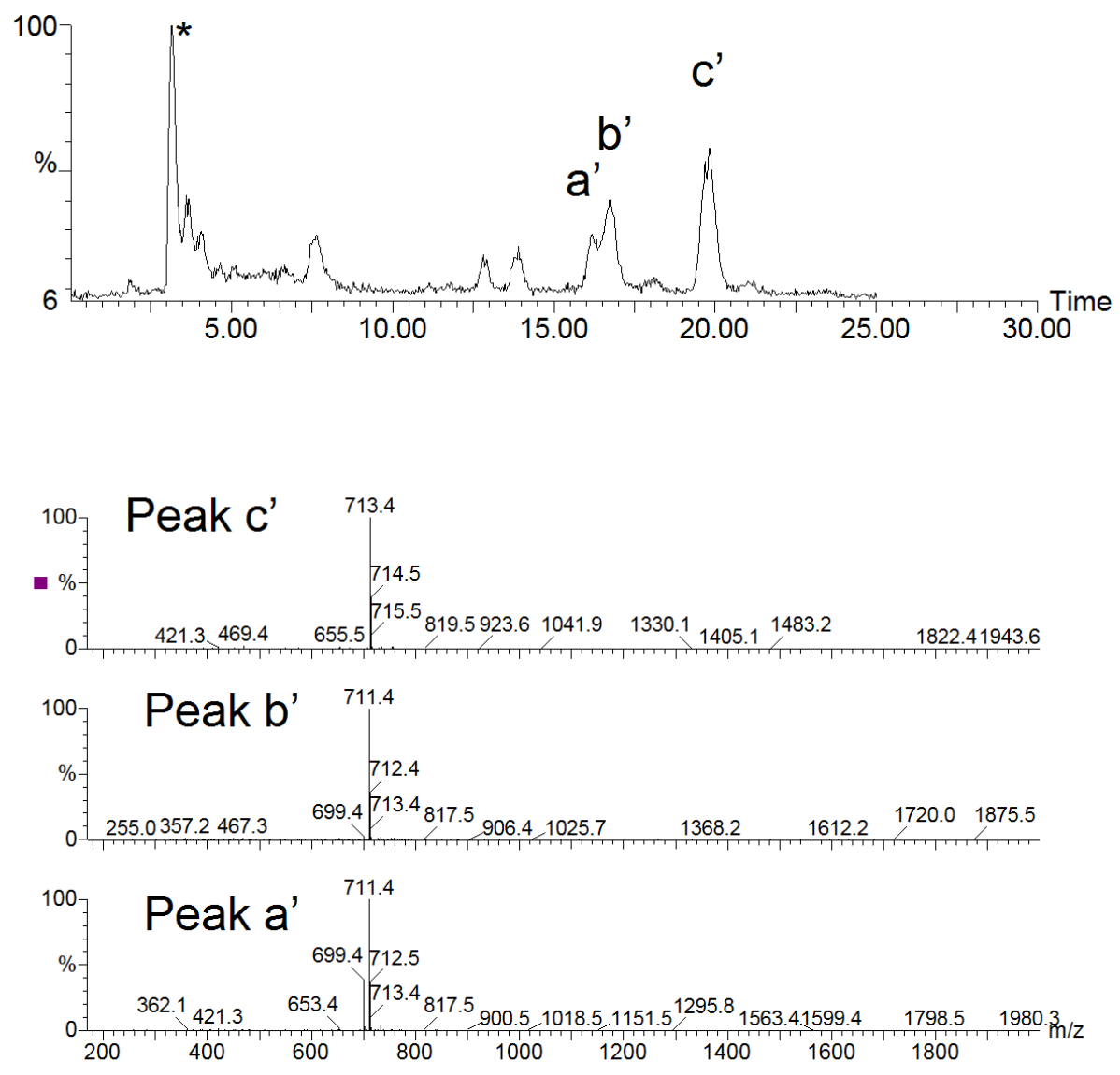

Figure S-1b. Total Ion chromatography (TIC) of unreacted residual sophorolipids recovered from ROMP reaction of lactonic sophorolipids (top), and Mass spectra of the major peaks, a', b' and c', in the TIC (bottom). These peaks are assigned same as peak a, b, and c in Figure S1-a, which are 17-L-([2'-O- $\beta$-D-glucopyranosyl- $\beta$-D-glucopyranosyl]oxy)-9-octadecenoic acid 1'4'"-lactone 6',6' -diacetate, 18-L-([2'-O- $\beta$-D-glucopyranosyl- $\beta$-D-glucopyranosyl $]$ oxy)-9octadecenoic acid 1'-4',-lactone 6',6',-diacetate, and 17-L-([2'-O- $\beta$-D-glucopyranosyl- $\beta$-Dglucopyranosyl]oxy)-octadecanoic acid 1'-4',-lactone 6', 6''-diacetate, respectively. The relative intensities of these peaks are different significantly from those in Figure S-1a. The peak marked as $*$ in the TIC was identified as poly(ethylene oxide) contamination. 

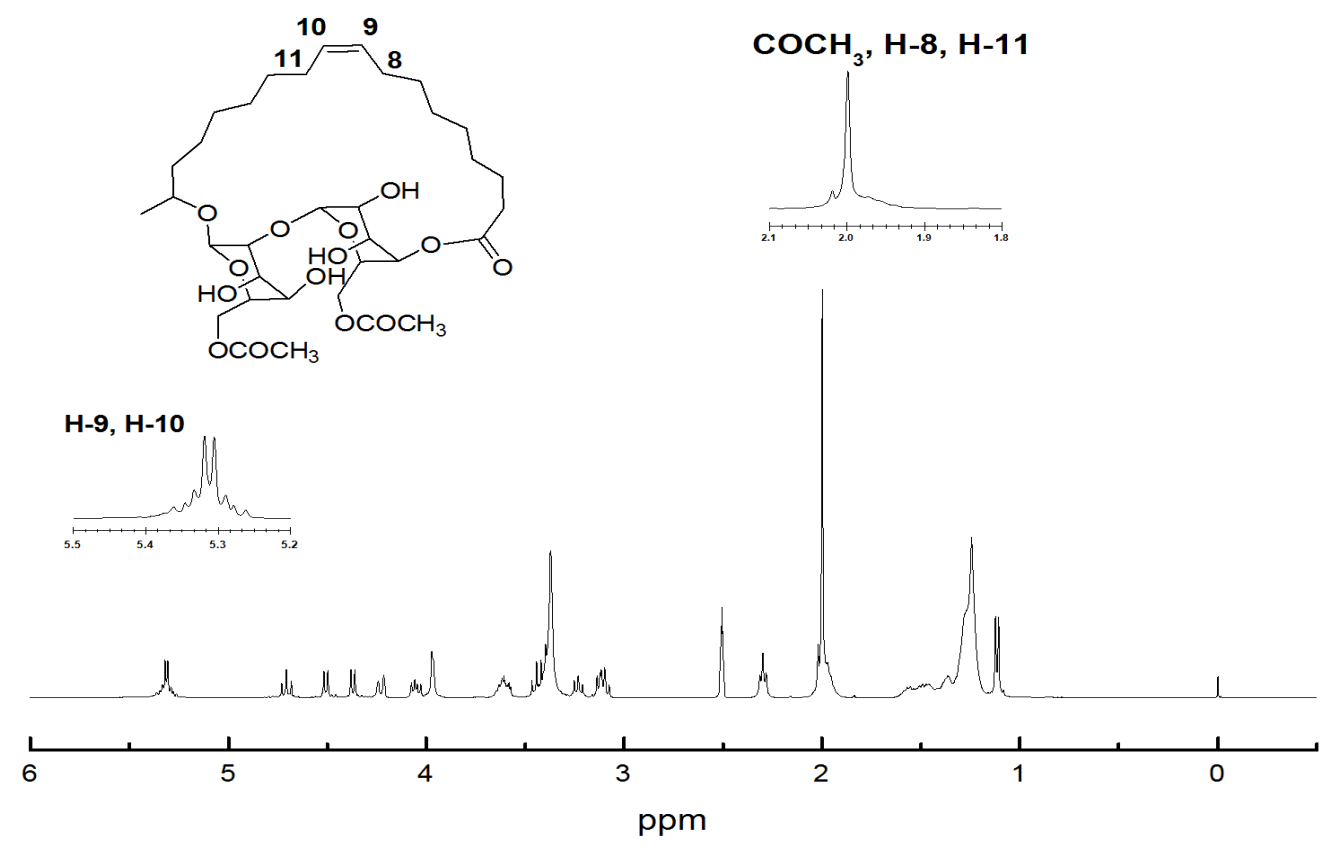

Figure S-2a. ${ }^{1} \mathrm{H}-\mathrm{NMR}$ (400 MHz, $d_{6}$-DMSO with $0.5 \% \mathrm{D}_{2} \mathrm{O}$ as solvent) of purified lactonicform sophorolipids.

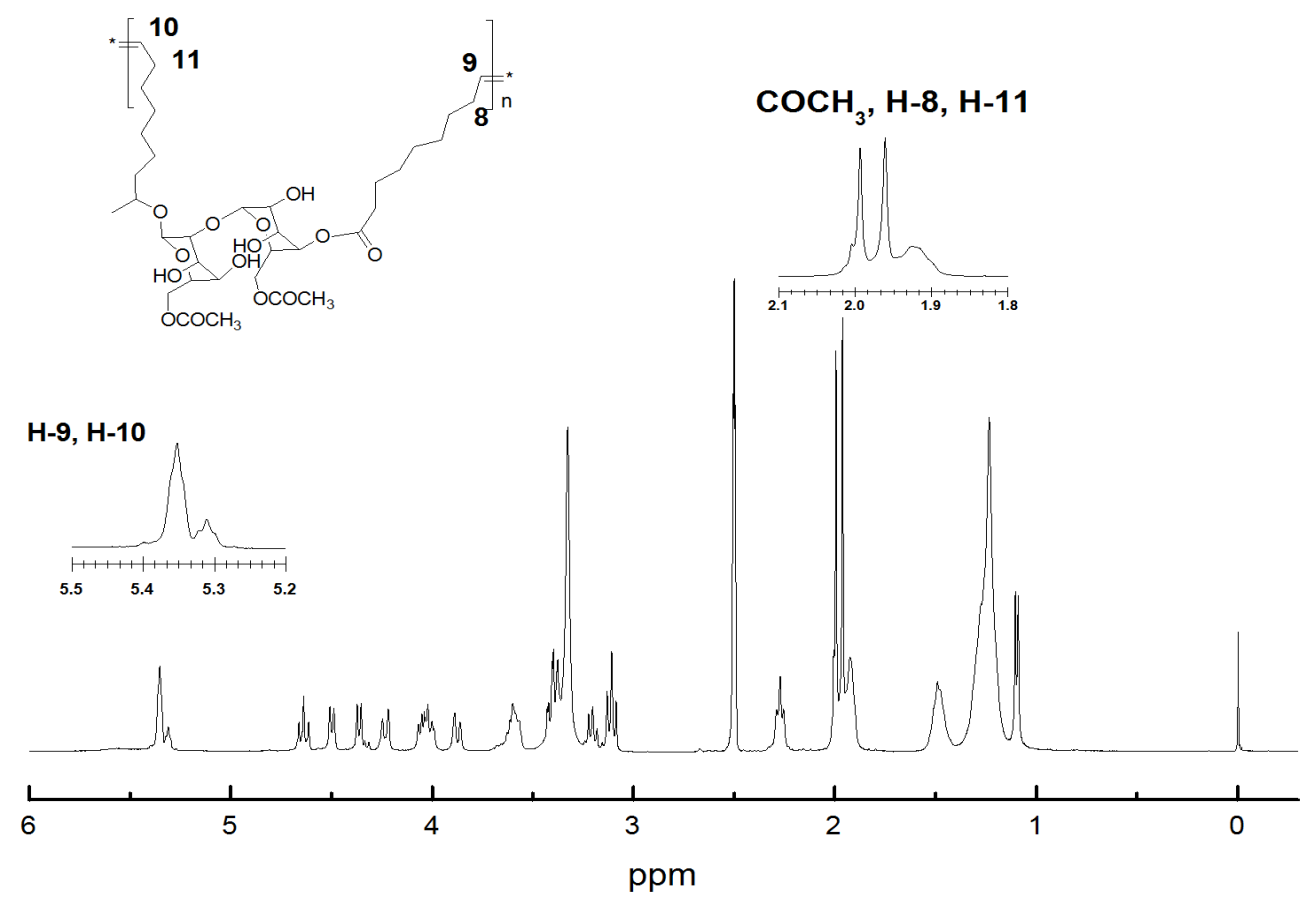

Figure S-2b. ${ }^{1} \mathrm{H}-\mathrm{NMR}$ (400 MHz, $\mathrm{d}_{6}$-DMSO with $0.5 \% \mathrm{D}_{2} \mathrm{O}$ as solvent) of polymer formed from metathesis polymerization of lactonic sophorolipid. 


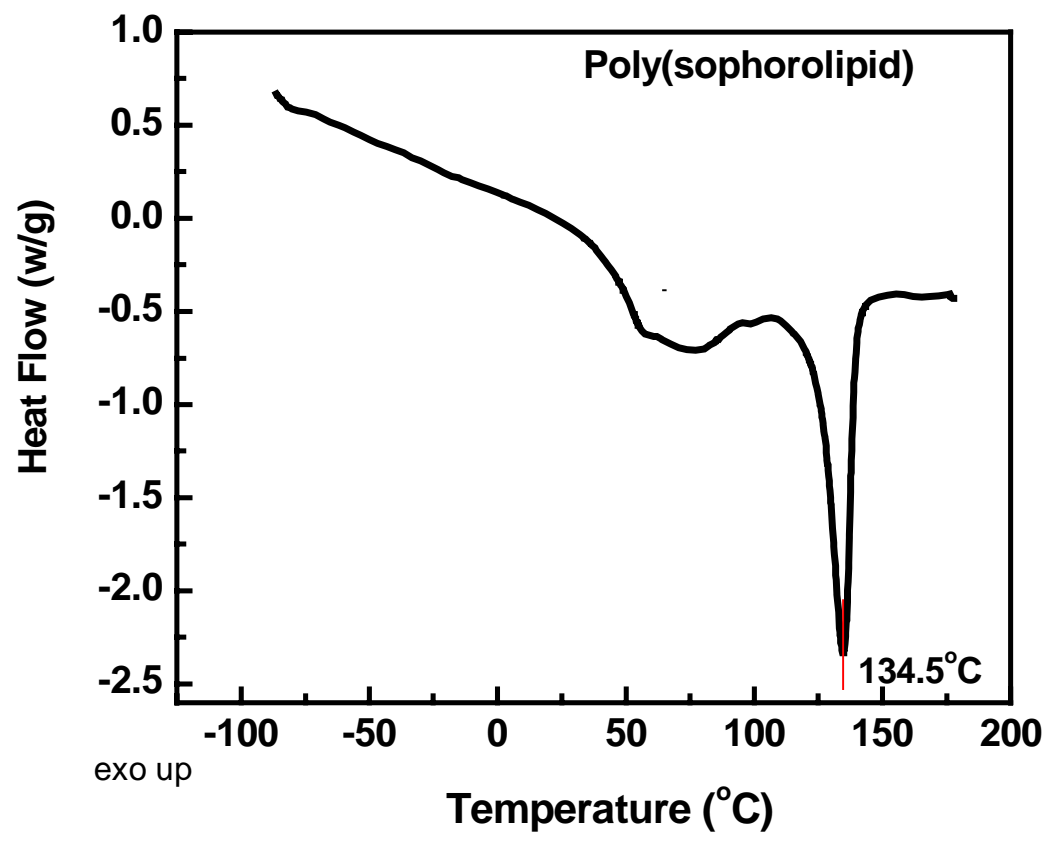

Figure S-3. DSC thermogram of poly(sophorolipid) recorded during the first heating scan at a heating rate of $10^{\circ} \mathrm{C} / \mathrm{min}$. 